\title{
Erratum: On the existence of almost periodic solutions of some dissipative second order differential equations
}

\author{
J. O. C. EzBrLo
}

[Ann. Mat. Pura Appl. (IV) Vol. IXV (1964), 389.406]

In $\$ 3$ of the above paper it was claimed (see Part (i) of the main Lemma) that the operator $T_{0}$ introduced in $\$ 2$ is completely continuous. This claim is now known to be untrue. As for the arguments adduced in $\$ 3$ for the complete continuity of $T_{0}$ the main slip occurs at the point where Ascour's theorem was invoked in order to establish the compactness of a certain sequence of functions. Ascoli's theorem gives uniform convergence only when the sequence of functions concerned is defined over a compact interval, and not on the whole real line as was the case for the sequence of functions discussed in $\S 3$.

The possibility is being currently explored of remedying the situation by working, for instance, with other function spaces. In the meantime the main question to which the paper is addressed, namely to find out what conditions are essential for the existence, only, of almost periodic solutions of the differential equation

$$
\ddot{x}+f(x) \dot{x}+g(x)=p(t)
$$

remains an open one. Theorem 3 of the paper and the corollary following it are unaffected. 